\title{
Le Comité consultatif national sur la prévention et le contrôle des infections (CCN-PCI)
}

\author{
T Ogunremi ${ }^{1 *}, \mathrm{~K}$ Dunn ${ }^{1}, \mathrm{~L}$ Johnston ${ }^{2}, \mathrm{~J} \mathrm{Embree}^{3}$, au nom du Comité consultatif national sur la \\ prévention et le contrôle des infections (CCN-PCl)
}

\section{Résumé}

Cet article décrit le travail du Comité consultatif national sur la prévention et le contrôle des infections (CCN-PCI), une comité consultatif national établi de longue date, qui offre à l'Agence de la santé publique du Canada (ASPC) une expertise et des conseils en matière de prévention et de contrôle des maladies infectieuses dans les établissements de soins de santé canadiens. Créé à l'origine par Santé Canada sous le nom de Comité directeur chargé de l'élaboration du guide de prévention des infections, en 1992, ce comité consultatif fournit des conseils d'experts relatifs à l'élaboration de lignes directrices sur la prévention et le contrôle des infections (PCl) depuis plus de 25 ans.

Le CCN-PCl offre des conseils destinés à guider l'élaboration de lignes directrices complètes ou abrégées, de guides de consultation rapide et de lignes directrices provisoires (le plus souvent sur des agents pathogènes émergents) et maintient une étroite collaboration avec les programmes nationaux de surveillance des infections associées aux soins de santé de l'ASPC en vigueur dans les établissements de santé canadiens. Les professionnels de la section de la prévention et du contrôle des infections associées aux soins de santé de l'ASPC effectuent la recherche documentaire, l'extraction de données, la synthèse des données probantes, l'évaluation de la qualité de ces données (s'il y a lieu) et la rédaction scientifique en vue d'élaborer des lignes directrices. Compte tenu de la rareté des études observationnelles de grande qualité et des études cliniques permettant de guider les recommandations sur les agents pathogènes émergents, il est essentiel d'obtenir l'opinion d'experts pour interpréter des données probantes disponibles.
Affiliations

${ }^{1}$ Centre de la lutte contre les maladies transmissibles et les infections, Agence de la santé publique du Canada, Ottawa (Ontario)

2 Université Dalhousie, Halifax (Nouvelle-Écosse)

${ }^{3}$ Université du Manitoba, Winnipeg (Manitoba)

Note : La composition du comité est précisée à la toute fin du présent article

*Correspondance : phac.ipc. secretariat-pci.aspc@canada.ca

Citation proposée : Ogunremi T, Dunn K, Johnston L, Embree J, au nom du Comité consultatif national sur la prévention et le contrôle des infections. Le Comité consultatif national sur la prévention et le contrôle des infections (CCNI-PCI). Relevé des maladies transmissibles au Canada 2018;44(11):322-29. https//doi. org/10/14745/ccdr.v44i11a03f

Mots-clés : prévention et contrôle des infections, comité consultatif, lignes directrices fondées sur des données probantes, infections associées aux soins de santé, maladies infectieuses

\section{Introduction}

Les maladies infectieuses, dans la mesure où elles constituent une menace mondiale, requièrent un échange international des connaissances et une réponse nationale coordonnée. Depuis sa création en 2004, l'Agence de la santé publique du Canada (ASPC) fait preuve d'un leadership à l'échelle nationale en réponse aux menaces pour la santé publique. Pour ce faire, elle utilise une approche fondée sur des données probantes faisant intervenir l'excellence scientifique des conseils pertinents provenant d'organismes consultatifs externes. Ces organismes consultatifs externes sont un moyen pour l'ASPC de faire appel à des personnes à l'extérieur du gouvernement, qui possèdent des connaissances et une expertise précieuses à l'égard du processus d'élaboration des lignes directrices nationales de l'Agence.

Les organismes consultatifs externes sont établis pour épauler I'ASPC dans le cadre de l'élaboration de directives portant sur des questions précises, de nature médicale, scientifique, technique, politique ou programmatique, qui relèvent du mandat de l'Agence (1). Les organismes consultatifs externes bien connus de l'ASPC regroupent, entre autres, le Comité 
consultatif national de l'immunisation (CCNI) et le Comité consultatif de la médecine tropicale et de la médecine des voyages (CCMTMV) $(2,3)$. Cet article décrit le travail du Comité consultatif national sur la prévention et le contrôle des infections (CCN-PCl).

\section{Contexte}

Le Comité directeur chargé de l'élaboration du guide de prévention des infections a été établi à l'origine par Santé Canada, en 1992. Ce comité, qui a joué un rôle central au cours de l'épidémie de syndrome respiratoire aigu sévère (SRAS) en 2003, relève de I'ASPC depuis la création de l'Agence en 2004. En 2011, le nom du comité a été remplacé par celui de Groupe d'experts sur la prévention et le contrôle des infections. Plus tôt, en 2018, il a été décidé de faire de ce groupe d'experts un organisme consultatif externe. Cette transition a donné lieu à un changement de nom du groupe, qui est devenu le $\mathrm{CCN}-\mathrm{PCl}$, et à un changement dans la structure hiérarchique. Par le passé, le $\mathrm{CCN}-\mathrm{PCl}$ relevait à l'ASPC à travers de Directeur des programmes mais relève à présent de la vice-présidente de la Direction générale de la prévention et du contrôle des maladies infectieuses. Le mandat et les fonctions du comité demeurent les mêmes.

La transition du $\mathrm{CCN}-\mathrm{PCl}$, d'un groupe d'experts à un organisme consultatif externe, respecte la politique et la directive de I'ASPC prévues pour de tels comités (1). La nouvelle structure hiérarchique découlant de ce changement consolidera les liens du CCN-PCl avec les partenaires provinciaux et territoriaux par l'intermédiaire du Conseil des médecins hygiénistes en chef. De tels liens sont particulièrement précieux en situation d'urgence, lorsque l'application en temps opportun de nouvelles lignes directrices et d'énoncés de position de la Section de la prévention et du contrôle des infections de I'ASPC associées aux soins de santé est cruciale. En guise d'exemples d'interventions passées, mentionnons les conseils touchant la santé publique et de nature scientifique et clinique offerts rapidement à I'ASPC au cours de la pandémie de grippe H1N1 (2009) et de l'urgence de santé publique de portée internationale provoquée par le virus Ebola (de 2013 à 2016).

Le présent article vise à décrire le mandat et la composition du $\mathrm{CCN}-\mathrm{PCl}$, à définir la façon dont ce dernier coordonne ses activités avec d'autres programmes de l'ASPC, à présenter un aperçu du processus d'élaboration des lignes directrices et à fournir une liste des lignes directrices de I'ASPC en vigueur élaborées en consultation avec les experts du CCN-PCI.

\section{Mandat et composition}

Le CCN-PCl a pour mandat d'aider l'ASPC à promouvoir la santé publique, à prévenir et à contrôler les maladies infectieuses, à se préparer aux urgences de santé publique et à y réagir, à servir de carrefour pour la diffusion de l'expertise canadienne en santé publique, à appliquer la recherche et les développements internationaux aux programmes nationaux de santé publique, à renforcer la collaboration intergouvernementale en santé publique, et à faciliter des approches nationales dans le secteur de la politique et de la planification en santé publique - le tout, dans la mesure où il se rapporte aux infections associées aux soins de santé.

Pour guider ces activités, le CCN-PCl dispense des conseils d'experts au personnel du programme de prévention et de contrôle des infections associées aux soins de santé en ce qui concerne :

- I'élaboration de lignes directrices nationales en matière de $\mathrm{PCl}$ qui sont fondées sur des données probantes et destinées aux établissements de soins de santé (4)

- la prestation d'avis techniques et scientifiques à I'ASPC, en réponse à l'émergence ou la réémergence d'agents pathogènes et de maladies infectieuses qui représentent une menace pour la santé publique

- I'élaboration de stratégies visant à prévenir et à contrôler les infections associées aux soins de santé, la résistance aux antimicrobiens (RAM) et d'autres problèmes de santé publique au Canada faisant intervenir la prestation de services de santé

- la détermination des priorités de la recherche en matière d'infections associées aux soins de santé et de $\mathrm{PCl}$

Le CCN-PCI regroupe jusqu'à 15 membres, qui sont recrutés au cours d'un processus de nomination transparent et ciblé. Le nombre de membres peut être ajusté pour assurer un éventail approprié d'expertise et d'expérience, ainsi qu'une représentation géographique adéquate. Le comité compte aussi parmi ses membres des agents de liaison n'ayant pas droit de vote, qui agissent en tant que représentants de provinces et de territoires ou d'associations et d'industries, et qui expriment des opinions au nom de l'organisation qui les emploie. Ces agents ou membres de liaison appuient le CCN-PCl en lui apportant des connaissances et des compétences supplémentaires, en partageant de l'information à jour pertinente en provenance de leur organisation respective, ainsi qu'en examinant et en commentant les énoncés de position et les documents d'orientation du CCN-PCl.

Un appel à candidatures ou un avis sollicitant les soumissions de candidats désireux de faire partie du CCN-PCl est envoyé aux associations professionnelles concernées pour diffusion dans leur communauté de pratique. La sélection des membres du comité repose sur un ensemble de critères, parmi lesquels le leadership, la représentation géographique, les connaissances spécialisées et l'agrément dans des domaines d'activités susceptibles de guider l'élaboration de lignes directrices et la réponse à des problèmes émergents ayant trait aux infections associées aux soins de santé.

Le comité est actuellement composé de membres possédant des compétences dans divers domaines : maladies infectieuses, 
microbiologie médicale, prévention et contrôle des infections, santé publique, épidémiologie des soins de santé et santé au travail et (ou) hygiène du travail. Des groupes de travail, dirigés par un membre du CCN-PCl et composés de membres du CCN-PCl et d'autres personnes possédant une expertise pertinente en la matière, sont nommés pour mener à bien I'élaboration de chaque ligne directrice ou produit. Les groupes de travail font rapport au $\mathrm{CCN}-\mathrm{PCl}$ pendant la phase d'élaboration du produit et le processus d'approbation préalable à la publication.

\section{Interconnectivité avec d'autres programmes et produits de I'ASPC}

Le programme de prévention et de contrôle des infections associées aux soins de santé collabore étroitement avec d'autres programmes de l'ASPC ayant des intérêts ou des mandats connexes. Cela inclut le Programme canadien de surveillance des infections nosocomiales (PCSIN), qui est responsable de la surveillance nationale (taux et tendances) des infections associées aux soins de santé, notamment des agents pathogènes émergents dans les établissements de soins de santé canadiens, de même que le Système canadien de surveillance de la résistance aux antimicrobiens (SCSRA), dont le rôle est d'assurer la surveillance nationale de la RAM et I'utilisation des antimicrobiens à l'échelle du pays $(5,6)$. Le travail effectué par ces programmes et par d'autres programmes connexes éclaire les travaux entrepris par le programme de CCN-PCI (p. ex. révisions d'un document d'orientation existant sur la prévention et le contrôle de l'infection par le bacille à gram négatif résistant aux carbapénèmes dans les établissements de soins de santé et d'autres produits de $\mathrm{PCl}$ de la RAM). Ces produits liés à la RAM contribueront au leadership national de I'ASPC sur cette question, tout en assurant la cohérence et I'harmonisation des produits de I'ASPC déjà publiés sur les infections associées aux soins de santé et la RAM.

\section{Processus d'élaboration des lignes directrices}

L'élaboration de lignes directrices est une activité de longue-haleine qui exige beaucoup de ressources et qui impose l'établissement des priorités et la collaboration en continu pour maximiser les ressources disponibles. L'établissement des priorités tient compte de différents facteurs : I'urgence d'une proposition de ligne directrice, la portée du problème, la présence d'une menace ou de répercussions pour la santé publique (en particulier dans le cas de l'émergence ou de la réémergence d'agents pathogènes), les priorités de l'ASPC et du Gouvernement du Canada, les demandes ou besoins déterminés au niveau provincial ou territorial en faveur d'une perspective nationale ayant pour but de faciliter une approche coordonnée, les lacunes cernées et la disponibilité de lignes directrices internationales appropriées. En tant que groupe, les membres et agents de liaison qui composent le CCN-PCI offrent leur évaluation des lignes directrices publiées concernées, fournissent de l'information sur des documents pertinents en cours d'élaboration par d'autres organisations et ciblent des possibilités de collaboration.

Le personnel du programme de prévention et de contrôle des infections associées aux soins de santé assume la fonction de chef de projet responsable des activités d'élaboration des lignes directrices. Ces activités comprennent la recherche documentaire, l'extraction de données, la synthèse des données probantes, l'évaluation critique de ces données, la rédaction de lignes directrices provisoires fondées sur des données probantes et la prestation de services de secrétariat au CCN-PCl. Les lignes directrices élaborées, qui entrent généralement dans l'une des quatre catégories suivantes, sont d'une complexité et d'une portée variables : lignes directrices complètes, lignes directrices abrégées, guides de consultation rapide et lignes directrices provisoires (le plus souvent sur des agents pathogènes émergents). L'élaboration des lignes directrices les plus complètes est généralement réalisée grâce à des recherches dans la littérature révisée par des pairs et la littérature scientifique grise en utilisant un processus d'examen systématique (voir figure 1). L'élaboration d'autres documents peut être éclairée par une revue narrative ou une analyse du contexte fondée sur une recherche documentaire ciblée. Chaque ligne directrice ou document contient une description des méthodes ou de l'approche utilisées pour son élaboration. Une fois les lignes directrices publiées, s'il y a lieu, le programme de prévention et de contrôle des infections associées aux soins de santé collabore avec le CCN-PCl à l'examen pertinent de nouvelles données probantes et à la mise à jour des lignes directrices.

\section{Évaluation de la qualité des données probantes}

L'élaboration de lignes directrices fait intervenir l'extraction des données pertinentes tirées de l'examen et de la synthèse de la littérature, l'interprétation des données probantes et l'évaluation des données probantes existantes (le cas échéant). Certaines lignes directrices sont surtout descriptives et éclairées par l'opinion d'experts, compte tenu de l'absence de données probantes publiées. Les critères utilisés pour établir la qualité des données probantes sur lesquelles s'appuie la série de lignes directrices nationales sur la $\mathrm{PCl}$ sont précisés dans le tableau 1.

\section{Élaboration de recommandations et prestation d'opinions d'experts}

Dans la mesure du possible, les recommandations sont appuyées par des données probantes provenant de tableaux récapitulatifs élaborés dans le cadre d'une revue systématique ou narrative 
Figure 1 : Processus d'élaboration des lignes directrices de l'ASPC sur la prévention et le contrôle des infections associées aux soins de santé

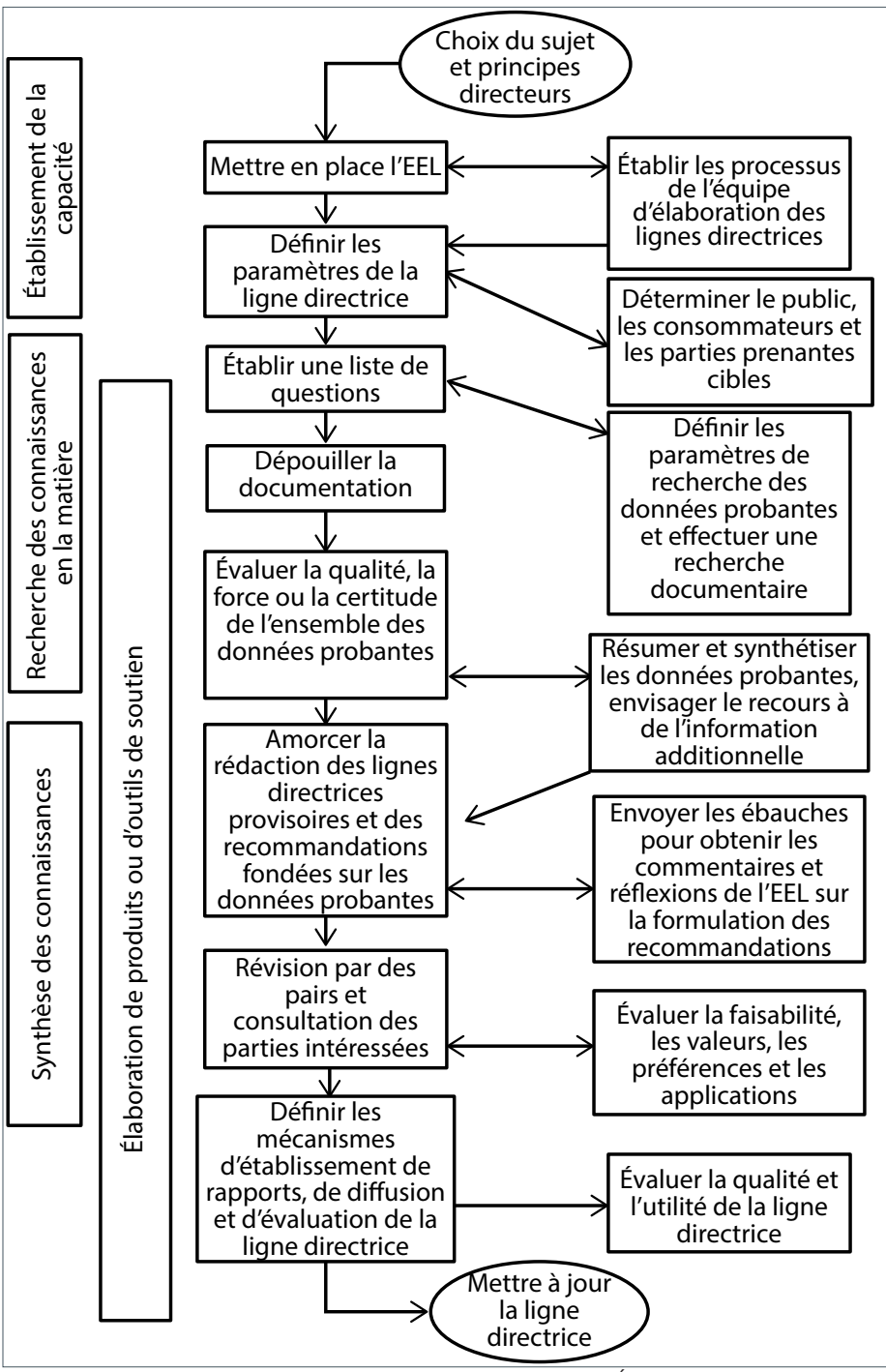

Abréviations : ASPC, Agence de la santé publique du Canada; EEL, Équipe d'élaboration de la ligne directrice

de la littérature. Pour des raisons d'éthique et de faisabilité, les études cliniques portant sur des questions courantes de prévention et de contrôle des infections sont pratiquement inexistantes, les études observationnelles sont limitées et les études descriptives ne fournissent pas de données probantes sur l'association causale. Par conséquent, l'opinion d'un expert est un aspect incontournable du processus d'élaboration de lignes directrices sur la prévention et le contrôle des infections associées aux soins de santé. L'opinion d'un expert est aussi essentielle pendant les premières phases d'une épidémie provoquée par un agent pathogène émergent, étant donné que les publications révisées par des pairs sont souvent limitées dans ces circonstances.
Tableau 1 : Critères d'évaluation de la qualité des données probantes servant de base aux lignes directrices sur la prévention et le contrôle des infections dans les milieux de soins ${ }^{a}$

\begin{tabular}{|c|c|c|}
\hline $\begin{array}{l}\text { Solidité des } \\
\text { données } \\
\text { probantes }\end{array}$ & $\begin{array}{l}\text { Qualité des } \\
\text { données } \\
\text { probantes }\end{array}$ & Critères \\
\hline \multirow[t]{2}{*}{ Élevée } & $\mathrm{Al}$ & $\begin{array}{l}\text { Données probantes directes provenant } \\
\text { d'une méta-analyse ou de multiples études à } \\
\text { modèle fort de haute qualité, avec résultats } \\
\text { cohérents }\end{array}$ \\
\hline & All & $\begin{array}{l}\text { Données probantes directes provenant de } \\
\text { multiples études à modèle fort de qualité } \\
\text { moyenne, avec résultats cohérents } \\
\text { OU } \\
\text { Au moins une étude à modèle fort étayée } \\
\text { par de multiples études à modèle modéré } \\
\text { de haute qualité, avec résultats cohérents } \\
\text { OU } \\
\text { Au moins une étude à modèle fort de qualité } \\
\text { moyenne étayée par une extrapolation de } \\
\text { multiples études à modèle fort de haute } \\
\text { qualité, avec résultats cohérents }\end{array}$ \\
\hline \multirow[t]{2}{*}{ Moyenne } & $\mathrm{BI}$ & $\begin{array}{l}\text { Données probantes directes provenant de } \\
\text { multiples études à modèle modéré de haute } \\
\text { qualité, avec résultats cohérents } \\
\text { OU } \\
\text { Extrapolation de multiples études à } \\
\text { modèle fort de haute qualité, avec résultats } \\
\text { cohérents }\end{array}$ \\
\hline & BII & $\begin{array}{l}\text { Données probantes directes provenant } \\
\text { de l'association d'études à modèle fort ou } \\
\text { modéré de qualité élevée ou moyenne, avec } \\
\text { une tendance claire, mais certains résultats } \\
\text { incohérents } \\
\text { OU } \\
\text { Extrapolation de multiples études à modèle } \\
\text { fort de qualité moyenne ou d'études à } \\
\text { modèle modéré de qualité élevée ou } \\
\text { moyenne, avec résultats cohérents } \\
\text { OU } \\
\text { Une étude à modèle fort étayée par de } \\
\text { multiples études à modèle faible de qualité } \\
\text { élevée ou moyenne, avec résultats cohérents }\end{array}$ \\
\hline \multirow[t]{2}{*}{ Faible } & $\mathrm{Cl}$ & $\begin{array}{l}\text { Données probantes directes provenant de } \\
\text { multiples études à modèle faible de qualité } \\
\text { élevée ou moyenne, avec résultats cohérents } \\
\text { OU } \\
\text { Extrapolation de l'association d'études à } \\
\text { modèle fort ou modéré de qualité élevée ou } \\
\text { moyenne, avec résultats incohérents }\end{array}$ \\
\hline & $\mathrm{CII}$ & $\begin{array}{l}\text { Étude de faible qualité, quel que soit le } \\
\text { modèle } \\
\text { OU } \\
\text { Résultats contradictoires, quel que soit le } \\
\text { modèle } \\
\text { OU } \\
\text { Études de série de cas ou exposés de cas } \\
\text { OU } \\
\text { Opinion d'un expert }\end{array}$ \\
\hline
\end{tabular}

Les recommandations en ce qui concerne les pratiques en santé publique s'appuient aussi sur l'épidémiologie des soins de santé, la surveillance et l'analyse des enjeux et tendances en matière de $\mathrm{PCl}$, ainsi que sur les commentaires des parties prenantes et 


\section{Tableau 2 : Infections associées aux soins de santé - lignes directrices sur la prévention et le contrôle des infections et autres documents publiés connexes}

\begin{tabular}{|c|c|c|}
\hline Sujet & Titre (année écoulée) & $\begin{array}{l}\text { Date de } \\
\text { publication/ } \\
\text { révision }\end{array}$ \\
\hline \multicolumn{3}{|c|}{ Documents complets } \\
\hline \multirow[t]{4}{*}{$\begin{array}{l}\text { Pratiques de } \\
\text { base }\end{array}$} & $\begin{array}{l}\text { Pratiques de base et précautions } \\
\text { additionnelles visant à prévenir la } \\
\text { transmission des infections dans les } \\
\text { milieux de soins } 2013 \text { (8) }\end{array}$ & 5 septembre 2014 \\
\hline & $\begin{array}{l}\text { Outils d'enseignement et } \\
\text { d'évaluation des pratiques de base } \\
\text { et des précautions additionnelles } \\
2013 \text { (9) }\end{array}$ & 5 septembre 2014 \\
\hline & $\begin{array}{l}\text { Affiche : Aidez à réduire la } \\
\text { propagation de la résistance } \\
\text { aux antimicrobiens - Suivez les } \\
\text { recommandations concernant les } \\
\text { pratiques de base dans les milieux } \\
\text { où des soins de santé sont dispensés } \\
2016(10)\end{array}$ & 26 mai 2016 \\
\hline & $\begin{array}{l}\text { Pratiques en matière d'hygiène des } \\
\text { mains dans les milieux de soins } 2012 \\
\text { (11) }\end{array}$ & 5 septembre 2014 \\
\hline $\begin{array}{l}\text { Infections } \\
\text { professionnelles }\end{array}$ & $\begin{array}{l}\text { La prévention et la lutte contre les } \\
\text { infections professionnelles dans le } \\
\text { domaine de la santé } 2002 \text { (12) }\end{array}$ & $\begin{array}{l}\text { Mars } 2002 \text { (en cours } \\
\text { de révision) }\end{array}$ \\
\hline $\begin{array}{l}\text { Infections } \\
\text { transmissibles } \\
\text { par le sang }\end{array}$ & $\begin{array}{l}\text { Compte rendu de la Conférence de } \\
\text { concertation sur les professionnels } \\
\text { de la santé infectés : Risque de } \\
\text { transmission des pathogènes à } \\
\text { diffusion hématogène (13) }\end{array}$ & $\begin{array}{l}\text { Juillet } 1998 \text { (en cours } \\
\text { de révision)a }\end{array}$ \\
\hline Pneumonie & $\begin{array}{l}\text { Guide de prévention de la } \\
\text { pneumonie associée aux soins de } \\
\text { santé } 2010 \text { (14) }\end{array}$ & 2010 \\
\hline \multirow[t]{2}{*}{ Endoscopie } & $\begin{array}{l}\text { Lignes directrices pour la prévention } \\
\text { et le contrôle des infections } \\
\text { transmises par les appareils souples } \\
\text { d'endoscopie digestive et de } \\
\text { bronchoscopie } 2011 \text { (15) }\end{array}$ & 10 février 2011 \\
\hline & $\begin{array}{l}\text { Avis : Pratiques recommandées pour } \\
\text { la prévention d'infections liées à des } \\
\text { endoscopies } 2016 \text { (16) }\end{array}$ & 24 mai 2016 \\
\hline \multicolumn{3}{|c|}{ Documents ciblés } \\
\hline $\begin{array}{l}\text { Bacille à } \\
\text { gram négatif } \\
\text { résistant aux } \\
\text { carbapénèmes }\end{array}$ & $\begin{array}{l}\text { Lignes directrices : Mesures } \\
\text { de prévention et de contrôle } \\
\text { des infections à l'intention des } \\
\text { travailleurs de la santé dans tous les } \\
\text { établissements de soins de santé : } \\
\text { Bacille gram négatif résistant aux } \\
\text { carbapénèmes } 2010 \text { (17) }\end{array}$ & $\begin{array}{l}3 \text { avril } 2012 \text { (en cours } \\
\text { de révision) }\end{array}$ \\
\hline \multirow[t]{2}{*}{$\begin{array}{l}\text { Clostridium } \\
\text { difficile }\end{array}$} & $\begin{array}{l}\text { Infection à Clostridium difficile: } \\
\text { Lignes directrices sur la prévention } \\
\text { et le contrôle des infections pour la } \\
\text { gestion dans les établissements de } \\
\text { soins actifs } 2013 \text { (18) }\end{array}$ & 11 janvier 2013 \\
\hline & $\begin{array}{l}\text { Infection à Clostridium difficile: } \\
\text { Lignes directrices sur la prévention } \\
\text { et le contrôle des infections pour la } \\
\text { gestion dans les établissements de } \\
\text { soins de longue durée } 2013 \text { (19) }\end{array}$ & 12 juillet 2013 \\
\hline $\begin{array}{l}\text { Maladie de } \\
\text { Creutzfeldt- } \\
\text { Jakob }\end{array}$ & $\begin{array}{l}\text { Guide de prévention : La maladie } \\
\text { de Creutzfeldt-Jakob classique au } \\
\text { Canada } 2007 \text { (20) }\end{array}$ & 1er novembre 2007 \\
\hline
\end{tabular}

Tableau 2 : (suite) Infections associées aux soins de santé - lignes directrices sur la prévention et le contrôle des infections et autres documents publiés connexes

\begin{tabular}{|c|c|c|}
\hline Sujet & Titre (année écoulée) & $\begin{array}{l}\text { Date de } \\
\text { publication/ } \\
\text { révision }\end{array}$ \\
\hline \multicolumn{3}{|c|}{ Documents ciblés (suite) } \\
\hline $\begin{array}{l}\text { Maladie de } \\
\text { Creutzfeldt- } \\
\text { Jakob (suite) }\end{array}$ & $\begin{array}{l}\text { La maladie de Creutzfeldt-Jakob } \\
\text { classique au Canada } 2002 \text { (21) }\end{array}$ & Novembre 2002 \\
\hline \multirow[t]{2}{*}{$\begin{array}{l}\text { Mycobacterium } \\
\text { tuberculosis et } \\
\text { autres espèces }\end{array}$} & $\begin{array}{l}\text { Chapitre } 15 \text { : Normes } \\
\text { canadiennes pour la lutte } \\
\text { antituberculeuse } 7 \text { e édition - La } \\
\text { prévention et la lutte contre la } \\
\text { transmission de la tuberculose dans } \\
\text { les milieux de soins de santé et } \\
\text { d'autres milieux } 2014 \text { (22) }\end{array}$ & 17 février 2017 \\
\hline & $\begin{array}{l}\text { Infections à Mycobacterium chimaera } \\
\text { chez les patients en phase : Aperçu } \\
\text { (23) }\end{array}$ & 4 mai 2017 \\
\hline \multirow[t]{2}{*}{$\begin{array}{l}\text { Grippe } \\
\text { saisonnière }\end{array}$} & $\begin{array}{l}\text { Grippe saisonnière - Lignes } \\
\text { directrices pour la prévention et } \\
\text { le contrôle des infections pour la } \\
\text { gestion dans le contexte des soins à } \\
\text { domicile } 2012 \text { (24) }\end{array}$ & 5 décembre 2012 \\
\hline & $\begin{array}{l}\text { Lignes directrices : Mesures de } \\
\text { prévention et de contrôle des } \\
\text { infections à l'intention des travailleurs } \\
\text { de la santé dans les établissements } \\
\text { de soins actifs et les établissements } \\
\text { de soins de longue durée - Grippe } \\
\text { saisonnière } 2010 \text { (25) }\end{array}$ & 20 décembre 2012 \\
\hline \multicolumn{3}{|c|}{ Infections émergentes } \\
\hline \multirow[t]{3}{*}{$\begin{array}{l}\text { Maladie à virus } \\
\text { Ebola }\end{array}$} & $\begin{array}{l}\text { Mesures de prévention et de } \\
\text { contrôle des infections : soins } \\
\text { préhospitaliers et transport terrestre } \\
\text { des patients chez qui la maladie } \\
\text { à virus Ebola est soupçonnée ou } \\
\text { confirmée (26) }\end{array}$ & 25 juin 2018 \\
\hline & $\begin{array}{l}\text { Groupe de travail d'experts en } \\
\text { prévention et en contrôle des } \\
\text { infections: Conseils relatifs aux } \\
\text { mesures de prévention et de contrôle } \\
\text { pour la maladie à virus Ebola dans les } \\
\text { milieux de soins (27) }\end{array}$ & 25 juin 2015 \\
\hline & $\begin{array}{l}\text { Groupe de travail d'experts en } \\
\text { prévention et en contrôle des } \\
\text { infections : Conseils relatifs à la } \\
\text { gestion des déchets associés à la } \\
\text { maladie à virus Ebola dans les milieux } \\
\text { de soins } 2015(28)\end{array}$ & 6 mai 2015 \\
\hline CoV-SRMO & $\begin{array}{l}\text { Lignes directrices sur la prévention } \\
\text { et le contrôle du coronavirus du } \\
\text { syndrome respiratoire du Moyen- } \\
\text { Orient (CoV-SRMO) dans les } \\
\text { établissements de soins actifs } 2016 \\
\text { (29) }\end{array}$ & 17 mai 2016 \\
\hline \multicolumn{3}{|c|}{ Autres documents } \\
\hline \multirow[t]{2}{*}{$\begin{array}{l}\text { Évaluation } \\
\text { critique }\end{array}$} & $\begin{array}{l}\text { Lignes directrices pour la prévention } \\
\text { et le contrôle des infections: Trousse } \\
\text { d'outils de l'évaluation critique } 2014 \\
\text { (30) }\end{array}$ & 11 mars 2015 \\
\hline & $\begin{array}{l}\text { Trousse d'outils de l'évaluation } \\
\text { critique pour l'évaluation de plusieurs } \\
\text { types de données probantes ( } 7 \text { ) }\end{array}$ & 7 septembre 2017 \\
\hline
\end{tabular}

Abréviation : CoV-SRMO, coronavirus du syndrome respiratoire du Moyen-Orient

a Un document fondé sur des données probantes qui remplacera ce document de consensus est en cours d'élaboration 
des partenaires provinciaux ou territoriaux. Les recommandations du $\mathrm{CCNPCl}$ viennent compléter les activités provinciales et territoriales et tiennent compte des lois, règlements et politiques des administrations fédérale, provinciales, territoriales et municipales. Le tableau 2 contient la liste des lignes directrices et d'autres publications élaborées par le programme de prévention et de contrôle des infections associées aux soins de santé, en consultation ou en collaboration avec un ou plusieurs membres du CCN-PCI (1).

\section{Conclusion}

À titre de comité consultatif externe, le CCN-PCl poursuit le travail accompli depuis 25 ans sous d'autres noms en fournissant des avis d'experts en vue de l'élaboration des lignes directrices nationales du CCN-PCI. La rigueur et les méthodes utilisées pour élaborer ces lignes directrices s'améliorent sans cesse, tout comme les possibilités de collaboration internationale ainsi que d'échange et de mobilisation des connaissances.

Le CCN-PCl est engagé à consolider ses liens avec les autres programmes de l'ASPC et ses partenaires externes et à informer le réseau fédéral-provincial-territorial élargi de la santé publique sur les questions d'IPC. Cela est important non seulement pour les affaires courantes, mais aussi pour les problèmes émergents qui menacent la santé publique et pourraient avoir une incidence sur les établissements de santé canadiens. Le cas échéant, le $\mathrm{CCN}-\mathrm{PCl}$ pourra fournir une interprétation d'experts sur les données probantes disponibles sur les agents pathogènes émergents et, au besoin, élaborer rapidement des lignes directrices fondées sur des données probantes en matière de protection et de contrôle des infections.

\section{Déclaration des auteurs}

A. J. - Conceptualisation, méthodes, rédaction de l'ébauche initiale, examen et édition

K. D. - Conceptualisation, supervision, rédaction, révision et édition

L. J. - Conceptualisation, rédaction, révision et édition

J. E. - Conceptualisation, rédaction, révision et édition

\section{Conflit d'intérêts}

Aucun.

\section{Collaborateurs}

Les auteurs tiennent à souligner les infatigables contributions de tous les membres du Comité consultatif national sur la prévention et le contrôle des infections (anciens et actuels) et leur engagement envers la prévention et le contrôle des maladies infectieuses au Canada :
Membres du CCN-PCI : Joanne Embree (présidente), Kathleen Dunn (secrétariat de la haute direction); Molly Blake, Gwen Cerkowniak, Maureen Cividino, Nan Cleator, Della Gregoraschuk, Bonnie Henry, Jennie Johnstone, Matthew Muller, Heidi Pitfield, Patsy Rawding, Patrice Savard, Stephanie Smith, Jane Stafford

Anciens membres du comité : Lynn Johnston (ancienne présidente et membre, 1996-2016), Lindsay Nicolle (ancienne présidente et membre, 1992-2006) et Kathleen Dunn (ancienne coprésidente); Sandra Boivin, Julie Carbonneau, John Conly, Brenda Dyck, John Embil, Karin Fluet, Charles Frenette, Colleen Hawes, Agnes Honish, Linda Kingsbury, Dany Larivée, Mary Leblanc, Anne Matlow, Catherine Mindorff, Dorothy Moore, Donna Moralejo, Deborah Norton, Shirley Paton, Diane Phippen, Pierre St. Antoine, Filomena Pietrangelo, Sandra Savery, JoAnne Seglie, Paul Sockett, Geoffrey Taylor, Mary Vearncombe, Cathie Walker, Dick Zoutman

Organisations-membres de liaison : Agrément Canada (AC), Association des infirmières en prévention des infections (AIPI), Association des médecins microbiologistes infectiologues du Québec (AMMIQ), Association pour la microbiologie médicale et I'infectiologie Canada (AMMI-Canada), Association canadienne en retraitement des dispositifs médicaux (ACRDM), Institut canadien d'information sur la santé (ICIS), Association médicale canadienne (AMC), Association des infirmières et infirmiers du Canada (AIIC), Association canadienne des infirmières et infirmiers en santé du travail (ACIIST), Institut canadien pour la sécurité des patients (ICSP), Association canadienne de normalisation (CSA), SoinsSantéCAN, Prévention et contrôle des infections $(\mathrm{PCl})$ - Canada, Les infirmières de l'Ordre de Victoria du Canada (VON Canada), Centres pour le contrôle et la prévention des maladies (Centers for Disease Control and Prevention, CDC)

Programme du CCN-PCl de l'ASPC (ancien et actuel) : Kathy Dunn (gestionnaire) Andrea Coady, Frédéric Bergeron, Katherine Defalco, Caroline Desjardins, Jennifer Kruse, Fanie Lalonde, Toju Ogunremi, Laurie O’Neil, Adina Popalyar, Christine Weir

\section{Remerciements}

Les auteurs aimeraient remercier Caroline Desjardins pour le formatage des tableaux, figures et références, ainsi que Hayley Watt pour la compilation de la liste des membres (anciens et actuels) du Comité consultatif national sur la prévention et le contrôle des infections. Les auteurs tiennent aussi à remercier Andrea Coady, Margaret Bodie, Katherine Defalco et Adina Popalyar pour la révision du document final. 


\section{Financement}

Le Comité consultatif national sur la prévention et le contrôle des infections au Canada est financé par l'Agence de la santé publique du Canada.

\section{Références}

1. Agence de la santé publique du Canada. Agence de la santé publique du Canada politique sur la gestion des organismes consultatifs externes de 2011 - Sommaire. Ottawa: SPC [mise à jour le 31 août 2012]. https://www.canada.ca/ fr/public-health/corporate/mandate/about-agency/ external-advisory-bodies/policy.html

2. Agence de la santé publique du Canada. Comité consultatif national de l'immunisation. À propos du CCNI. Ottawa: Gouvernement du Canada. https://www.canada.ca/fr/ sante-publique/services/immunisation/comite-consultati f-national-immunisation-ccni.html

3. Agence de la santé publique du Canada. Qu'est-ce que le CCMTMV?. Ottawa: Gouvernement du Canada. https:// www.canada.ca/fr/sante-publique/services/sante-voyageurs/ est-ccmtmv.html

4. Centre de la lutte contre les maladies transmissibles et les infections, Agence de la santé publique du Canada. Les infections aux soins de santé : Série des Guides de prévention des infections. Ottawa: Gouvernement du Canada. https://www.canada.ca/fr/sante-publique/ services/maladies-infectieuses/infections-nosocomialesprofessionnelles.html

5. Centre de la lutte contre les maladies transmissibles et les infections. Programme canadien de surveillance des infections nosocomiales (PCSIN). Ottawa: Agence de la santé publique du Canada. http://www.phac-aspc.gc.ca/nois-sinp/ survprog-fra.php

6. Agence de la santé publique du Canada. Actualités sur les maladies infectieuses: Système canadien de surveillance de la résistance aux antimicrobiens - faits saillants du rapport de 2017. Relevé des maladies transmissibles au Canada 2017;43(11):281. https://www.canada.ca/fr/sante-publique/ services/rapports-publications/releve-maladie s-transmissibles-canada-rmtc/numero-mensuel/2017-43/ rmtc-volume-43-11-2-novembre-2017/systeme-canadie n-surveillance-resistance-antimicrobiens-faits-saillan ts-rapport-2017.html

7. Moralejo D, Ogunremi T, Dunn K. Trousse d'outils de l'évaluation critique pour l'évaluation de plusieurs types de données probantes. Relevé des maladies transmissibles au Canada 2017;43(9):199-205. DOI

8. Agence de la santé publique du Canada. Pratiques de base et précautions additionnelles visant à prévenir la transmission des infections dans les milieux de soins (2013). https://www. canada.ca/fr/sante-publique/services/maladies-infectieuses/ infections-nosocomiales-professionnelles/pratiques-bas e-precautions-additionnelles-visant-a-prevenir-transmi ssion-infections-milieux-soins.html
9. Agence de la santé publique du Canada. Outils d'enseignement et d'évaluation des pratiques de base et des précautions additionnelles (2013). https://www. canada.ca/fr/sante-publique/services/maladies-infectieuses/ infections-nosocomiales-professionnelles/outils-enseignemen t-evaluation-pratiques-base-precautions-additionnelles.html

10. Agence de la santé publique du Canada. Affiche: Aidez à réduire la propagation de la résistance aux antimicrobiens - Suivez les recommandations concernant les pratiques de base dans les milieux où des soins de santé sont dispensés. https://www.canada.ca/fr/sante-publique/services/ publications/medicaments-et-produits-sante/aidez-reduir e-propagation-resistance-aux-antimicrobiens.html

11. Agence de la santé publique du Canada. Pratiques en matière d'hygiène des mains dans les milieux de soins (2012). https://www.canada.ca/fr/sante-publique/ services/maladies-infectieuses/infections-nosocomialesprofessionnelles/pratiques-matiere-hygiene-main s-milieux-soins.html

12. Santé Canada. La prevention et la lute contre les infections professionnelles dans le domaine de la santé. RMTC 2002;28SI:1-287(sous révisions). http://publications.gc.ca/ collections/collection_2016/aspc-phac/HP3-1-28-S1-fra.pdf

13. Agence de la santé publique du Canada. Compte rendu de la Conférence de concertation sur les professionnels de la santé infectés: Risque de transmission des pathogènes à diffusion hématogène. RMTC 1998 Jul;24 Suppl 4:i-iii, 1-25; i-iii, 1-28 (sous révisions). http://publications.gc.ca/ collections/collection_2016/aspc-phac/HP3-1-24-S4-fra.pdf

14. Agence de la santé publique du Canada. Guide de prévention de la pneumonie associée aux soins de santé (2010). http://publications.gc.ca/collections/collection_2012/ aspc-phac/HP40-54-2010-fra.pdf

15. Agence de la santé publique du Canada. Lignes directrices pour la prévention et le contrôle des infections transmises par les appareils souples d'endoscopie digestive et de bronchoscopie. https://www.canada. $\mathrm{ca} / \mathrm{fr} / \mathrm{sante}$-publique/services/maladies-infectieuses/ infections-nosocomiales-professionnelles/lignes-directrice s-prevention-controle-infections-transmises-appareilssouples-endoscopie-digestive-bronchoscopie.html

16. Agence de la santé publique du Canada. Avis: Pratiques recommandées pour la prévention d'infections liées à des endoscopies. https://www.canada.ca/fr/sante-publique/ services/maladies-infectieuses/infections-nosocomialesprofessionnelles/pratiques-recommandee s-prevention-infections-liees-a-endoscopies.html

17. Agence de la santé publique du Canada. Lignes directrices: Mesures de prévention et de contrôle des infections à l'intention des travailleurs de la santé dans tous les établissements de soins de santé : Bacille Gram négatif résistant aux carbapénèmes (sous révisions). https://www. canada.ca/fr/sante-publique/services/maladies-infectieuses/ infections-nosocomiales-professionnelles/lignes-directrice s-mesures-prevention-controle-infections-a-intentiontravailleurs-sante-tous-etablissements-soins-sante.html

18. Agence de la santé publique du Canada. Infection à Clostridium Difficile : Lignes directrices sur la prévention 
et le contrôle des infections pour la gestion dans les établissements de soins actifs. https://www.canada. $\mathrm{ca} / \mathrm{fr} /$ sante-publique/services/maladies-infectieuses/ infections-nosocomiales-professionnelles/infectiona-clostridium-difficile-lignes-directrices-infections-etablissem ents-soins-actifs.html

19. Agence de la santé publique du Canada. Infection à Clostridium difficle - Lignes directrices sur la prévention et le contrôle des infections pour la gestion dans les établissements de soins de longue durée. https://www. canada.ca/fr/sante-publique/services/maladies-infectieuses/ infections-nosocomiales-professionnelles/infectiona-clostridium-difficile-lignes-directrices-infections-etablissem ents-longue-duree.html

20. Agence de la santé publique du Canada. La maladie de Creutzfeldt-Jakob classique au Canada: Guide de consultation rapide. https://www.canada.ca/ $\mathrm{fr} /$ sante-publique/services/maladies-infectieuses/ infections-nosocomiales-professionnelles/ maladie-creutzfeldt-jakob/guide-prevention-infections.html

21. Santé Canada. La maladie de Creutzfeldt-Jakob classique au Canada. Guide de prévention des infections. RMTC 2002;28S5:1-93. http://publications.gc.ca/collections/ collection_2016/aspc-phac/HP3-1-28-S5-fra.pdf

22. Agence de la santé publique du Canada. Normes Canadiennes pour la lutte antituberculeuse $7^{e}$ édition; Chapitre 15 - La prévention et la lutte contre la transmission de la tuberculose dans les milieux de soins de santé et d'autres milieux. https://www.canada.ca/fr/sante-publique/ services/maladies-infectieuses/normes-canadienne s-lutte-antituberculeuse-7e-edition/edition-11.html

23. Ogunremi T, Taylor G, Johnston L, Amaratunga K, Muller M, Coady A, Defalco K, Dunn K, Johnstone J, Smith S, Embree J, Henry B, Stafford J, au nom du groupe de travail d'experts en prévention et en contrôle des infection. Infections à Mycobacterium chimaera chez les patients en phase post-opératoire exposés à des échangeurs thermiques : Un aperçu. Relevé des maladies transmissibles au Canada 2017;43(5):119-26. DOI

24. Agence de la santé publique du Canada. Grippe saisonnière - Lignes directrices pour la prévention et le contrôle des infections pour la gestion dans le contexte des soins à domicile. https://www.canada.ca/fr/sante-publique/ services/maladies-infectieuses/infections-nosocomialesprofessionnelles/grippe-saisonniere-lignes-directri ces-prevention-controle-infections-gestion-contexte-soi ns-a-domicile.html

25. Agence de la santé publique du Canada. Lignes directrices : Mesures de prévention et de contrôle des infections à l'intention des travailleurs de la santé dans les établissements de soins actifs et les établissements de soins de longue durée - Grippe saisonnière (2010). https://www.canada. $\mathrm{ca} / \mathrm{fr} /$ sante-publique/services/maladies-infectieuses/ infections-nosocomiales-professionnelles/lignes-directrice s-mesures-prevention-controle-infections-a-intentiontravailleurs-sante-etablissements-soins-actifs-etabli ssements-soins-longue.html

26. Agence de la santé publique du Canada. Mesures de prévention et de contrôle des infections : Soins préhospitaliers et transport terrestre des patients chez qui la maladie à virus Ebola est soupçonnée ou confirmée. https://www.canada.ca/ $\mathrm{fr} /$ sante-publique/services/maladies/maladie-virusebola/pour-professionnels-sante-maladie-virus-ebola/ ebola-mesures-transport-patients.html

27. Agence de la santé publique du Canada. Groupe de travail d'experts en prévention et en contrôle des infections: Conseils relatifs aux mesures de prévention et de contrôle pour la maladie à virus Ebola dans les milieux de soins. https://www.canada.ca/fr/sante-publique/services/ maladies-infectieuses/fievres-hemorragiques-virales/ groupe-travail-experts-prevention-controle-infections-cons eils-relatifs-mesures-prevention-controle-maladie-v irus-ebola-milieux-soins-1.html

28. Agence de la santé publique du Canada. Groupe de travail d'experts en prévention et en contrôle des infections: Conseils relatifs à la gestion des déchets associés à la maladie à virus Ebola dans les milieux de soins. https://www. canada.ca/fr/sante-publique/services/maladies-infectieuses/ fievres-hemorragiques-virales/groupe-travail-experts-pre vention-controle-infections-conseils-relatifs-gesti on-dechets-associes-maladie-virus-ebola-milieux-soins.html

29. Agence de la santé publique du Canada. Lignes directrices sur la prévention et le contrôle du coronavirus du syndrome respiratoire du Moyen-Orient (CoV-SRMO) dans les établissements de soins actifs. https://www.canada.ca/ $\mathrm{fr} /$ sante-publique/services/publications/maladies-etaffections/lignes-directrices-prevention-et-controle-corona virus-syndrome-respiratoire-moyen-orient-cov-s rmo-etablissements-soins-actifs.html

30. Agence de la santé publique du Canada. Lignes directrices pour la prévention et le contrôle des infections: Trousse d'outils de l'évaluation critique (2014). https://www.canada. $\mathrm{ca} / \mathrm{fr} /$ sante-publique/services/maladies-infectieuses/ infections-nosocomiales-professionnelles/lignes-directrice s-prevention-controle-infections-trousse-outils-evaluationcritique.html 